\title{
Commentary Will Viewing Overeating as Compulsive Lead to Novel Pharmacological Interventions?
}

\author{
Kristin N Javaras ${ }^{*, 1,2}$ \\ 'Division of Women's Mental Health, McLean Hospital, Belmont, MA, USA; ${ }^{2}$ Department of Psychiatry, Harvard Medical School, Boston, MA, USA
}

Neuropsychopharmacology (2017) 42, 1373-1374; doi:I0.1038/npp.2017.29; published online I March 2017

Overeating-consuming food in excess of one's needs-has become a commonplace occurrence in many parts of the world, especially the United States. For some individuals, however, overeating becomes highly recurrent and (in the words of Moore et al, 2017) 'pathological,' causing psychological distress, impairment in daily functioning, or physical health problems. Although there are many forms of pathological overeating, the best studied is binge eating. Clinically, binge eating is operationalized as consuming an unusually large amount of food during a short period of time, accompanied by a sense of loss of control. Binge-eating behavior is a defining characteristic of bulimia nervosa $(\mathrm{BN})$, a psychiatric disorder in which recurrent episodes of binge eating are accompanied by inappropriate weight control behaviors such as vomiting. Binge-eating behavior can also occur in anorexia nervosa (AN), a psychiatric disorder characterized by persistent behaviors (eg, extreme dietary restriction) to maintain low body weight. Further, an additional $4.0 \%$ of US men and women report having experienced binge-eating episodes twice a week or more outside the context of AN or BN (Hudson et al, 2007). Individuals who experience recurrent binge eating in the absence of inappropriate or extreme weight control behaviors can receive a diagnosis of binge-eating disorder (BED), provided the binge-eating behavior causes them psychological distress. BED is the most common eating disorder in the United States, and it is associated with significant functional impairment, especially in the social domain (Kessler et al, 2013). Although not all individuals with BED have high levels of adiposity (and vice versa), individuals with BED are more likely to have a body mass index in the obese range. Further, BED may confer risk for development of metabolic syndrome over and above its association with obesity. Cognitive-behavioral therapy and psychotropic medications (eg, second-generation antidepressants, lisdexamfetamine) are efficacious treatments for BED (Brownley et al, 2016), but many individuals do not respond entirely or at all, and

*Correspondence: Dr KN Javaras, McLean Hospital, I I 5 Mill Street, Mail Stop 117, Belmont, MA 02478, USA, Tel: +1 617855 2302, E-mail: kjavaras@mclean.harvard.edu

Accepted article preview online 6 February 2017 relapse can occur after cessation of treatment. Thus, there is considerable room for advances in the treatment of BED, as well as other forms of pathological overeating.

To this end, Moore et al posit that certain forms of pathological overeating, including binge eating in $\mathrm{BED}$, are compulsive in nature and can be usefully modeled by three, non-mutually-exclusive elements (or domains) thought to characterize drug addiction: (1) habitual overeating, (2) overeating to relieve a negative emotional state, and (3) overeating despite aversive consequences. Each of these domains is instantiated via (overlapping) neurobiological substrates including the basal ganglia, the extended amygdala, and the prefrontal cortex, respectively.

The idea that pathological overeating-like drug addiction - can be driven by compulsion is not new. Peer-based support groups (eg, Overeaters Anonymous) have embraced the idea that overeating can be compulsive in nature. Individual clinical researchers have long noted that overeating behavior in BED (McElroy et al, 1994) and obesity (Mount et al, 1990) can resemble the compulsive behaviors observed in obsessive-compulsive disorders. Further, certain aspects of Moore et al's formulation of compulsive eating have been popular topics of research for decades (eg, overeating to relieve negative moods or stress). Over the past decade, however, the analogy of drug addiction has increasingly been applied to both obesity and BED, and more research has focused on examining the domains of compulsivity and underlying neurobiological substrates described by Moore et al, aided on the clinical side by advances in neuroimaging. Likewise, research on 'food addiction,' a construct thought to overlap with BED and obesity, has grown, although not without debate regarding the construct itself and the extent of its applicability (eg, to BN; Fairburn, 2013, Chapter 7).

Moore et al add to these burgeoning areas of research by clearly describing elements of compulsive eating anchored in specific behavioral paradigms that can be applied to a wide variety of repetitive behaviors. For instance, outcome devaluation paradigms, which examine the extent to which behaviors persist even once the desired outcome (eg, enjoyment of food) has been devalued, can be used to operationalize habitual overeating. This focus on specific 
behavioral paradigms neatly sidesteps debates that ensue when terms with multiple interpretations or operationalizations (eg, addiction) are used to describe pathological eating by analogy to other phenomena. More importantly, focusing on specific behavioral paradigms provides both preclinical and clinical researchers with a concrete approach to examining pathological overeating in animal models and clinical populations, respectively.

It is an empirical question whether adopting these behavioral paradigms, and examining the neurobiological substrates underlying them, will advance our understanding of pathological overeating and ultimately lead to novel interventions. As always, the challenge for clinical researchers will be identifying the patient populations for whom the various elements of compulsive overeating are relevant. Some preliminary evidence supports the appropriateness of these behavioral paradigms, and the involvement of their underlying neurobiological substrates, in BED and obesity, although it is important to bear in mind that obesity and even BED are heterogeneous. Of course, even models that appear to fit a phenomenon at a behavioral or a neural level may not prove useful at understanding or altering it if they do not capture the truly causal variables driving that phenomenon. As an illustrative example, some individuals with AN experience recurrent episodes of binge eating, which can feel compulsive and are often very distressing for the individual. Although these episodes cannot objectively be described as aversive in consequence (if anything, bingeeating is adaptive in the context of starvation), they could meet behavioral criteria for the other domains of compulsivity and involve some of the same neural systems (eg, basal ganglia) implicated in drug addiction. However, focusing on compulsive overeating behavior in AN would be like focusing on 'compulsive breathing' when someone is suffocating. The larger problem-and the one that needs to be addressed first-is dietary restriction. Although individuals with BED do not exhibit the degree of dietary restriction observed in $\mathrm{AN}$, many individuals with $\mathrm{BED}$ strive to dramatically reduce caloric intake and avoid certain (eg, highly palatable) foods. In BED as well as AN and BN, targeting excessive dietary restriction, whether actual or intended, can help to resolve binge eating by removing the physiological and psychological deprivation, respectively, that can drive it. However, for binge eating and other forms of pathological overeating that arise or continue outside the context of excessive dietary restriction, Moore et al's formulation of compulsive overeating may prove very helpful at generating novel insights and interventions.

\section{FUNDING AND DISCLOSURE}

KNJ's research is supported by McLean Hospital and by philanthropic support from The Phyllis and Jerome Lyle Rappaport Foundation, Nancy Dearman, and the Martini Family Foundation. She has received an honorarium from the National Institute on Aging for coauthoring a commissioned review. She also owns stock in Sanofi and Centene Corporation. The author declares no conflict of interest.

\section{ACKNOWLEDGMENTS}

The author would like to thank Dr James Hudson, Dr Christine Peat, and the Editor-in-Chief for insightful comments on a draft of this manuscript.

\section{REFERENCES}

Brownley KA, Berkman ND, Peat CM, Lohr KN, Cullen KE, Bann CM et al (2016). Binge-eating disorder in adults: a systematic review and meta-analysis. Ann Intern Med 165: 409-420.

Fairburn CG (2013). Overcoming Binge Eating, 2nd edn. Guilford Press: New York.

Hudson JI, Hiripi E, Pope Jr HG, Kessler RC (2007). The prevalence and correlates of eating disorders in the National Comorbidity Survey Replication. Biol Psychiatry 61: 348-358.

Kessler RC, Berglund PA, Chiu WT, Deitz AC, Hudson JI, Shahly V et al (2013). The prevalence and correlates of binge eating disorder in the World Health Organization World Mental Health Surveys. Biol Psychiatry 73: 904-914.

McElroy SE, Phillips KA, Keck PE Jr. (1994). Obsessive compulsive spectrum disorder. J Clin Psychiatry 55 (Suppl): 33-51.

Moore CF, Sabatino V, Koob GF, Cottone C. (2017). Pathological overeating: emerging evidence for a compulsivity construct. Neuropsychopharmacology (this issue).

Mount R, Neziroglu F, Taylor CJ (1990). An obsessive-compulsive view of obesity and its treatment. J Clin Psychol 46: 68-78. 